\section{Le parasite Leishmania à l'ère de la post-génomique}

Marc Ouellette, Martin Olivier, Sachiko Sato, Barbara Papadopoulou espèces, responsable de différentes pathologies et représente une cause importante de morbidité et de mortalité en médecine humaine et vétérinaire. Ce parasite intracellulaire est transmis par l'intermédiaire de la mouche des sables chez un hôte vertébré, où, après différentiation, il se multiplie dans les macrophages. Le séquençage du génome de l'espèce Leishmania major est quasiment terminé. Ces données de séquences s'avèrent une source inestimable pour des études transcriptomiques et protéomiques qui permettent de mieux comprendre le processus de différentiation du parasite, son interaction avec la cellule hôte et sa capacité à résister aux traitements conventionnels. <

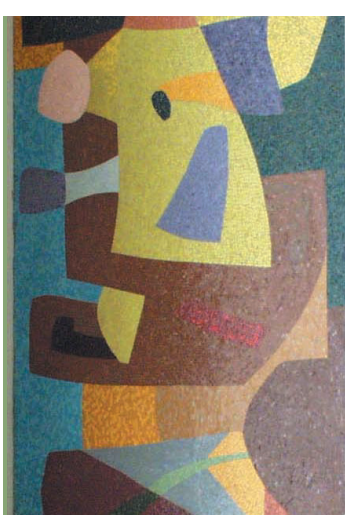

Le parasite

Les progrès récents en génomique et en protéomique ont revitalisé la recherche en microbiologie et parasitologie. Depuis la publication de la séquence du génome de la bactérie Haemophilus influenzae en 1995 [1], de nombreux génomes microbiens ont été séquencés. Fin 2002, on comptait déjà plus de 100 génomes microbiens complètement séquencés et plusieurs centaines dont le séquençage était en cours (http://wit.integratedgenomics.com/GOLD). Les parasites ne sont pas en reste comme en atteste le séquençage récemment publié du génome de Plasmodium falciparum, agent du paludisme [2] et celui, en cours, de plusieurs autres parasites. Nous nous intéresserons ici au séquençage du génome du parasite Leishmania. En effet, depuis 1990 la Faculté de Médecine de l'Université Laval a développé un pôle de recherche en parasitologie où s'effectue, sous les auspices d'un groupe des Instituts de Recherche en Santé du Canada (IRSC), une recherche intégrée sur le parasite Leishmania portant sur les interactions hôtes-pathogènes (y compris la co-infection avec le VIH), sur le processus développemental et sur la chimiorésistance du parasite au traitement.

\section{Leishmania}

Leishmania est un parasite protozoaire de l'ordre des kineto-

plastidae qui comprend entre autres Trypanosoma brucei, agent de la maladie du sommeil, et Trypanosoma cruzi, agent de la maladie de Chagas. Leishmania a un cycle de vie dimorphique. Chez la mouche des sables (Figure IB), principalement les espèces Lutzomyia et Phlebotomus pour l'immense majorité des cas de transmission à l'homme [3], on retrouve ce parasite sous une forme promastigote, d'abord procyclique non infectieuse, puis, après différenciation dans le tractus digestif de l'insecte, sous forme métacyclique et infectieuse. Le promastigote, de forme allongée et flagellée (Figure lA), est d'une grande mobilité. La transmission à l'homme est réalisée par piqûre par un insecte infecté. Une fois chez l'hôte, le parasite pénètre dans les macrophages (la transformation de promastigotes en amastigotes n'a pas encore été démontrée dans les cellules dendritiques) où le promastigote se trans- 
forme en amastigote. L'amastigote, de forme arrondie, n'a pas de flagelle développé et est peu mobile (Figure ID). L'infection se répand chez un individu par relargage/internalisation de macrophage à macrophage. Bien que l'infection par Leishmania soit de fait une maladie transmise à partir des animaux (zoonose), où I'homme est souvent un hôte facultatif, les preuves, dans certaines régions particulières, d'un cycle de transmission d'une personne à l'autre (anthroponotique) s'accumulent. Plusieurs espèces de Leishmania peuvent infecter l'homme et provoquer des manifestations cliniques différentes connues sous le nom de leishmanioses [4]. Leishmania major par exemple provoque des leishmanioses cutanées dont les lésions sont généralement, mais pas toujours, réversibles. D’autres espèces, comme le complexe Viannia, sont la cause de leishmanioses mucocutanées qui touchent les muqueuses et sont beaucoup plus débilitantes. Plus graves, les Leishmania du complexe donovani provoquent des leishmanioses viscérales qui sont souvent fatales en l'absence de traitement. Malgré une assez bonne corrélation entre espèce et pathologie, il existe des exceptions comme les cas de leishmanioses viscérales observées chez des soldats ayant participé à I'Opération Tempête du Désert et qui sont dues à $L$. tropica, normalement responsable de leishmanioses cutanées [5].

Leishmania est un parasite de vaste distribution géographique retrouvé en Asie, Afrique, Amérique du Sud et Centrale et en Europe, particulièrement autour du bassin méditerranéen. Les différentes espèces infectent plus de 15 millions de personnes et on dénombre deux millions de nouveaux cas chaque année [4]. II n'existe pas encore de vaccins efficaces pour prévenir la maladie et les tentatives de contrôle du vecteur sont pour l'instant restées sans succès. Le traitement des infections dues à ce parasite repose donc essentiellement sur la chimiothérapie. La pharmacopée disponible contre le Leishmania est limitée et le traitement prescrit en première intention est un composé à base d'antimoine pentavalent (SbV), une molécule utilisée depuis plus de 50 ans. Quelques traitements alternatifs sont disponibles, comme l'amphothéricine $B$ et la pentamidine, cependant plus toxiques. De plus, bien que l'amphothéricine B sous forme liposomiale ait un excellent index thérapeutique, elle est financièrement hors de portée pour la majorité des pays où la maladie est endémique [4]. La chimiorésistance à l'antimoine pentavalent est en progression constante et dans certaines régions de l'Inde, plus de la moitié des patients ne répondent plus à cette molécule. Les mécanismes de résistance sont en partie définis chez des souches où la résistance a été induite in vitro mais le mode de résistance chez les souches du terrain reste encore à préciser. Tous les aspects décrits précédemment, processus développemental, tropisme et pathologie, interaction avec la cellule hôte et chimiorésistance devraient largement profiter des nouvelles techniques de génomique, transcriptomique et protéomique qui se développent. La corrélation entre tropisme et pathologie est encore mal définie et doit naturellement faire intervenir des facteurs du parasite et de l'hôte. Une génomique comparative entre différentes espèces de Leishmania pourrait mettre en évidence la présence de gènes espèce-spécifiques impliqués dans ces diverses pathologies. L'étude globale (transcriptome et protéome) des interactions Leishmania-cellules hôtes pourrait par ailleurs donner des informations précieuses sur la réponse de l'hôte à l'infection et suggérer ainsi de nouvelles stratégies d'immunomodulation.

\section{Organisation et séquence génomique chez Leishmania}

Le projet de séquençage de Leishmania a pris forme lors d'une réunion à Rio de Janeiro en 1994. De cette réunion est né le Leishmania Genome Network, un consortium de laboratoires impliqués dans la cartographie, le séquençage et l'annotation des génomes et dans les tests fonc- 
tionnels qui en découlent. Un projet de séquençage a débuté avec la souche Leishmania major MHOM/IL/81/Friedlin. Son génome d'environ 35 Mb est distribué sur 36 chromosomes dont la taille varie de 0,3 à 2,8 Mb. II faut noter que certaines espèces de $L$. major n'ont que 34 ou 35 chromosomes [6] mais la complexité du génome est globalement conservée. Actuellement, plus de $34 \mathrm{Mb}$ de séquences ont été accumulées dans les banques de données. La séquence des chromosomes 1,3 , $4,5,15,24,25$ et 31 est terminée et le génome de Leishmania est régulièrement annoté (www.genedb.org).

L'analyse des données disponibles a révélé une densité de 1 gène/3,5 kb avec très peu de séquences répétées. On s'attend à approximativement 8000 gènes et un nombre un peu plus élevé de protéines, ce qui ne peut être dû à des phénomènes d'épissage alternatif, Leishmania étant dépourvu d'introns. Les premières analyses suggèrent que plus de la moitié des gènes seront uniques à Leishmania et qu'une proportion encore plus grande n'auront pas de fonction identifiée. Les gènes sont organisés de façon particulière puisqu'ils font partie de larges unités polycistroniques. Ainsi sur le chromosome 1, il y a une unité de 29 gènes sur un brin d'ADN et de 50 gènes sur l'autre brin [7]. Dans le cas du chromosome 1 , les unités polycistroniques sont convergentes mais pour d'autres chromosomes elles peuvent être divergentes ou encore avoir une combinaison d'unités convergentes et divergentes (Figure 2). Leishmania est un parasite qui a divergé tôt dans l'arbre phylogénétique des eukaryotes et son mode de régulation de l'expression des gènes présente des propriétés inédites. En effet, il ne semble pas exister de régulation au niveau de l'initiation de la transcription mais essentiellement une régulation post-transcriptionelle. Ainsi, de longs messagers contenant tous les gènes organisés en tandem sont modifiés par addition d'un mini-exon en 5' (par épissage en trans), et par polyadénylation en 3 ' (Figure 2). Tous les messagers de Leishmania ont ce mini-exon de 39 nucléotides à leur extrémité 5 ' et son addition est étroitement associée à la polyadénylation [8].

\section{Différenciation du parasite et survie intracellulaire}

Chez l'insecte vecteur, Leishmania se différencie, dans le tractus digestif, de la forme promastigote procyclique flagellée en la forme métacyclique infectieuse, qui migre ultimement dans la trompe (proboscide) de l'insecte et sera ainsi transmise à l'hôte lors d'un repas sanguin [3]. Le parasite pénètre dans les macrophages où il se différencie dans le phagolysosome (vacuole digestive) en la forme amastigote. Il est possible de faire croître en laboratoire les divers stades de différentiation de plusieurs espèces de Leishmania. Dans différents milieux de culture relativement complexes, pendant la phase de croissance logarithmique à $25^{\circ} \mathrm{C}$, le parasite est sous la forme promastigote. Quand la forme promastigote atteint la phase stationnaire, il y a transformation en forme métacyclique suivie de l'expression de marqueurs spécifiques [9]. Traditionnellement, la forme amastigote des parasites était recueillie à partir de culture de macrophages ou d'animaux infectés. Depuis quelques années, des conditions de culture en milieu stérile (milieu axénique) ont été réalisées qui permettent la multiplication de la forme amastigote de certaines espèces de Leishmania caractérisée par l'expression de marqueurs amastigotesspécifiques (Figure 1E). Les stimuli qui favorisent la transformation in vitro de promastigotes en amastigotes

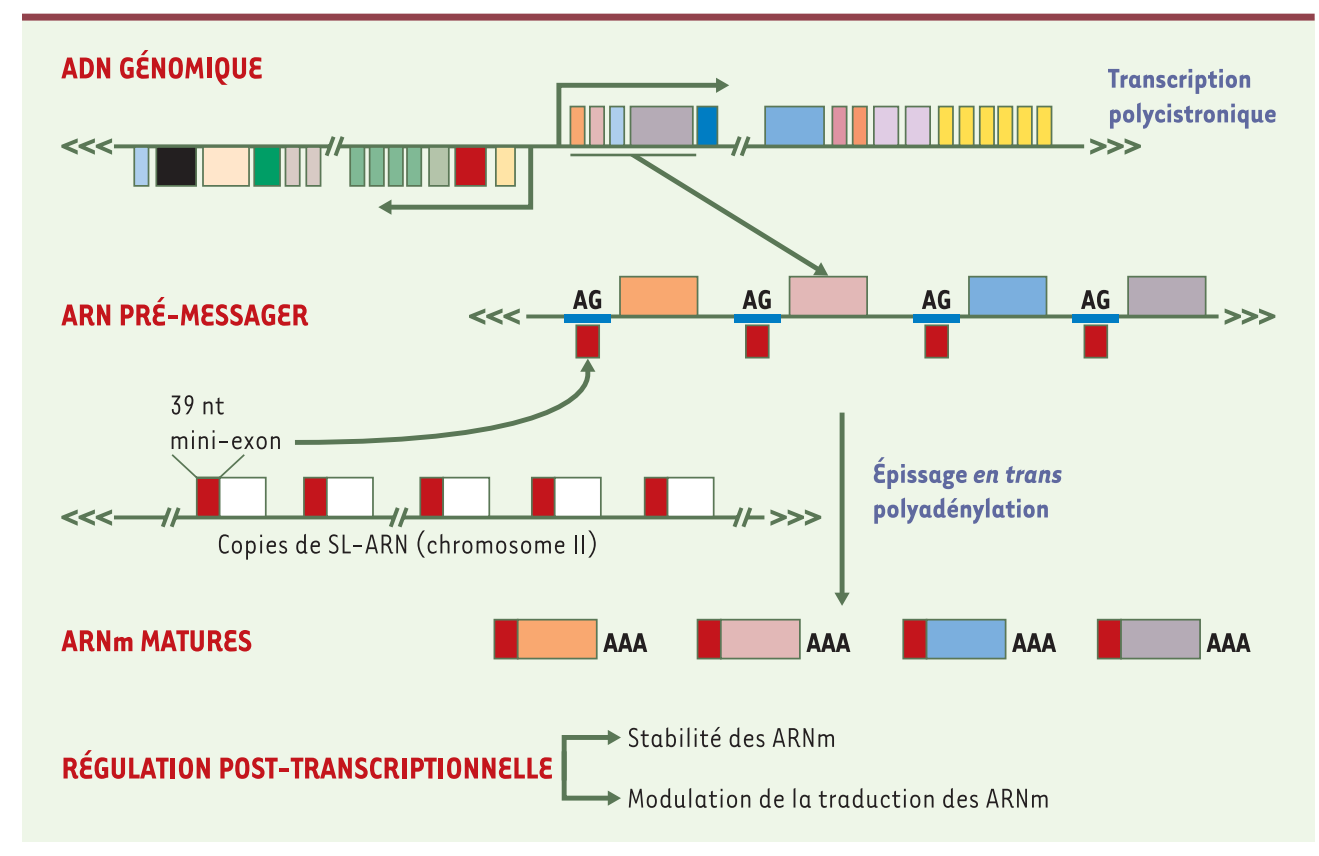

Figure 2. Organisation génique et maturation des messagers chez Leishmania. Les gènes de Leishmania sont organisés en unités polycistroniques. Ces unités sont co-transcrites en très grands pré-messagers qui sont maturés par l'addition d'un mini-exon en 5' (épissage en trans) et par polyadénylation en 3'. Les réactions d'épissage en trans et de polyadenylation sont couplées. SL-ARN, spliced leader ARN; nt, nucléotide. 
sont le pH acide et la température [10]. En effet, les promastigotes se trouvent naturellement chez la mouche des sables dans un milieu neutre et à une température de $25^{\circ} \mathrm{C}$, tandis qu'à l'intérieur du phagolysosome du macrophage de l'hôte, le parasite vit à $37^{\circ} \mathrm{C}$ et à $\mathrm{pH}$ acide. Cette possibilité de contrôler la multiplication des parasites à différents stades de leur cycle de vie a facilité les études qui visent à identifier des gènes exprimés différentiellement chez le parasite.

\section{Analyse transcriptomique}

Bien qu'il n'y ait pas, chez Leishmania, de régulation de l'expression des gènes au niveau transcriptionnel, plusieurs messagers semblent s'accumuler à des stades de développement spécifiques (Figure 1E). Les mécanismes impliqués sont peu connus mais pourraient faire intervenir une modulation de la stabilité des ARNm, soit par régulation de la polyadénylation soit par interaction avec des facteurs spécifiques [8]. Des techniques de criblage différentiel, ont permis d'isoler une vingtaine de gènes exprimés spécifiquement à l'un des stades de différentiation du parasite. Nos analyses récentes de criblage différentiel, réalisées sur des ADNc de promastigotes et d'amastigotes hybridés sur des filtres à haute densité, ont montré que quelques gènes sont différentiellement exprimés entre ces deux stades parmi lesquels des gènes codant pour une famille de protéines membranaires appelée les amastines [11, 12]. D'autres travaux utilisant des technologies apparentées ont également révélé l'expression de gènes spécifiques de ces stades de différentiation [13, 14]. Une technique qui gagne en popularité pour mesurer l'expression différentielle des gènes est celle des puces à ADN. Cette technique permet de déposer sur des lames de verre des milliers de sondes qui peuvent ensuite être hybridées simultanément avec des ADNc marqués par des nucléotides fluorescents provenant, par exemple, de deux stades différents de différentiation (Figure 3). Ce type de technique a déjà montré son efficacité pour de nombreux micro-organismes [15] et pour quelques parasites comme l'agent de la malaria [16] ou T. brucei [17]. Bien que le génome de Leishmania ne soit pas connu dans son intégralité, des puces ont été réalisées soit avec des fragments d'ADNc, soit avec des fragments aléatoires génomiques de $1 \mathrm{~kb}[18,19]$ et quelques leçons peuvent d'ores et déjà être tirées de leur utilisation. Tout d'abord, il a été possible de confirmer l'expression différentielle qui avait été prédite pour certains gènes, ce qui a validé la technique. Cependant, l'expression de seulement 1 à $2 \%$ des gènes s'est trouvée modulée par un facteur de 2 ou plus entre la forme promastigote et métacyclique [19]. Ce pourcentage est en deçà des changements observés entre la forme logarithmique et stationnaire par exemple chez $\varepsilon$. coli ou chez S. cerevisiae (environ 5\%) [20, 21]. Ceci peut s'expliquer par le mode particulier de régulation génétique de Leishmania (Figure 2) qui utiliserait préférentiellement une régulation de type traductionnel. Nous avons effectivement identifié dans la région 3' non traduite du messager de l'amastine une séquence qui gouverne l'efficacité de la traduction et ce spécifiquement au stade amastigote [22]. Une analyse in silico des $34 \mathrm{Mb}$ de séquences de Leishmania a révélé que cette séquence est très répandue et dans chaque cas étudié, contrôle la traduction des gènes spécifiquement au stade amastigote [22]. Les mécanismes impliqués dans ce nouveau mode de régulation de l'expression des gènes sont actuellement à l'étude. 


\section{Analyse protéomique}

Cette propension de Leishmania à exercer une régulation traductionnelle a motivé une analyse protéomique comparative entre la forme promastigote et amastigote. Dans une analyse initiale nous avons montré que plus de $5 \%$ des protéines sont différentiellement exprimées entre les deux stades de différenciation. Parmi celles-ci, nous avons identifié par spectrométrie de masse deux enzymes du cycle glycolytique dont l'expression est augmentée chez l'amastigote [23], ce qui est cohérent avec le mode de production de I'ATP qui, contrairement aux promastigotes, dépend principalement de la glycolyse chez les amastigotes. Par électrophorèse bidimensionnelle et en utilisant de nouveaux logiciels d'analyse, nous avons récemment réalisé une analyse des protéines solubles exprimées chez $L$. infantum (Figure 4). Plus de $6 \%$ des protéines exprimées le sont soit exclusivement soit préférentiellement dans la forme amastigote et un pourcentage similaire est spécifique du promastigote. L'analyse protéomique semble plus prometteuse que les puces à ADN pour identifier des gènes exprimés différentiellement. Les gels bidimensionnels présentent cependant plusieurs limitations, comme la sous-représentation des protéines membranaires ou des protéines faiblement exprimées. De nouvelles technologies isotopiques d'affinité [24] ou d'électrophorèse multidimensionnelle seront nécessaires [25] pour une meilleure représentation des protéomes. déjà la comparaison entre $P$. falciparum et $P$. yoelli a révélé une grande synthénie (conservation de l'organisation des gènes sur les chromosomes) pour les gènes métaboliques, mais au contraire d'importantes variations d'organisation pour les antigènes de surface et les molécules permettant l'évasion de la réponse de l'hôte [27]. Chez Leishmania, la comparaison du locus qui porte le transporteur de la bioptérine sur le chromosome 35 chez $L$. major et $L$. donovani a d'ailleurs montré une très grande synthénie génique [28, 29]. L’hybridation compétitive des puces à ADN de L. major avec, par exemple, de l'ADN marqué provenant de L. major et de L. donovani devrait permettre la détection de gènes potentiellement spécifiques de L. major (Figure 3). Des expériences similaires pourront être réalisées sur des puces portant le génome de L. donovani, afin d'identifier des gènes qui lui sont spécifiques. II sera particulièrement intéressant de comparer les gènes des espèces responsables de diverses pathologies entre eux mais aussi avec les gènes des espèces de Leishmania non pathogènes pour l'homme. Les molécules de surface du parasite comme le lipophosphoglycane (LPG), un glycolipide largement représenté à la surface du parasite, sont sans doute déterminantes dans les interactions avec la cellule hôte. La séquence des sucres substitués sur l'ossature du LPG est très différente selon les espèces de Leishmania. Par exemple, L. major contient

\section{Génomique comparative et interactions hôtes-pathogènes}

Les différentes espèces de Leishmania provoquent différentes pathologies et ce tropisme spécifique est à la fois dû à des propriétés intrinsèques aux différentes souches de Leishmania et aux interactions entre des facteurs du parasite et des facteurs de l'hôte. Par exemple, l'un des gènes phare amastigote spécifique chez L. donovani est le gène A2 [13]. Ce gène est tronqué chez $L$. major et des parasites $L$. major recombinants exprimant le gène $A 2$ de $L$. donovani ont un pouvoir de viscéralisation augmenté [26]. Une analyse de génomique comparative entre différentes espèces de Leishmania devrait permettre d'identifier les gènes caractéristiques de chaque espèce. Actuellement seul le séquençage du génome de $L$. major est en cours. Cependant, la technologie du séquençage s'améliorant, il est possible que le génome de L. donovani infantum dans un premier temps et de plusieurs autres espèces de Leishmania par la suite soient complètement séquencés. Le cas de plasmodium est dans ce contexte tout à fait exemplaire. Six espèces de Plasmodium sont en cours de séquençage (http://plasmodb.org/) et

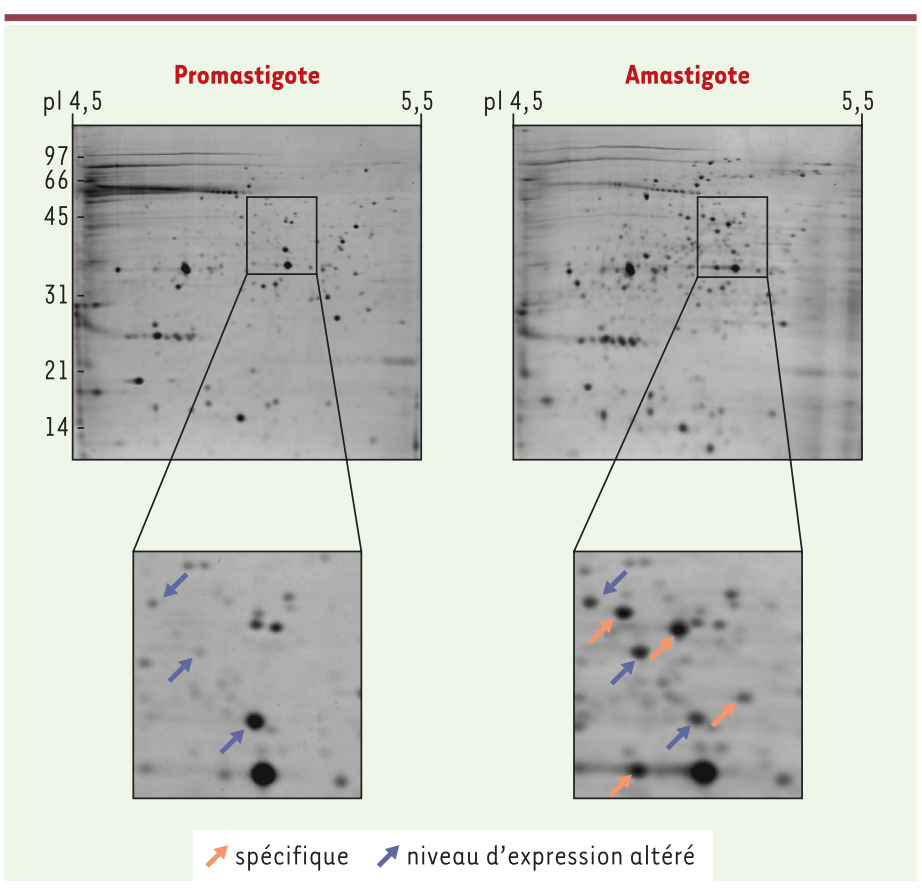

Figure 4. Expression différentielle des protéines de Leishmania donovani. Séparation par électrophorèse bidimensionnelle des protéines des formes promastigote et amastigote. 
du poly- $\beta$-galactose tandis qu'il n'y a pas de sucres substitués chez L. donovani [30]. Ces molécules pourraient être à l'origine des variations observées dans la réponse de l'hôte vis à vis des différentes espèces. Nous avons par exemple montré sur des modèles murins que L. major induit un recrutement cellulaire massif de leucocytes et la sécretion marquée de cytokines proinflammatoires, tandis que $L$. donovani ne provoque qu'une très légère réponse inflammatoire au site primaire d'infection [31]. Cette réponse inflammatoire très forte pourrait expliquer le maintien du parasite et le développement de lésions cutanées aux sites d'inoculation spécifiques de L. major.

Les LPG qui sont spécifiques de chaque espèce sont reconnus par des récepteurs de la cellule hôte appelés lectines. Nous avons récemment montré que la galectine-3, une lectine sécrétée par les macrophages et les cellules dendritiques activées par des molécules pro-

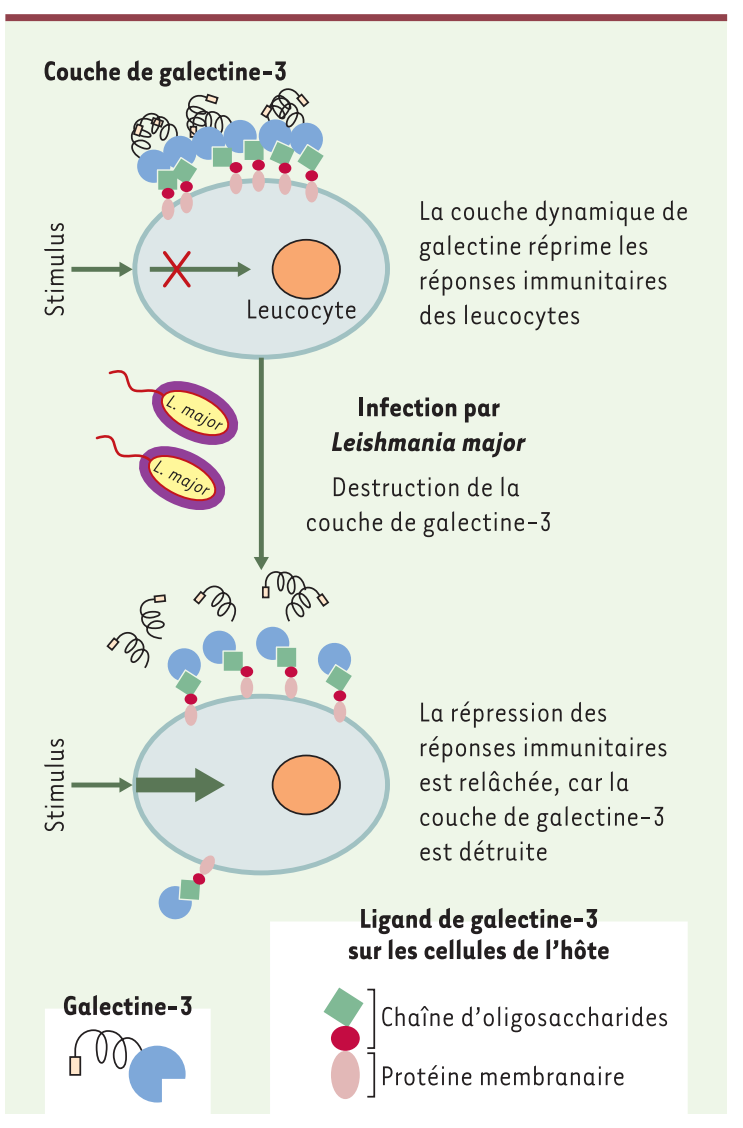

Figure 5. Rôle de la galectine 3 dans les interactions avec Leishmania major. Les lipophosphoglycanes (LPG) à la surface de $L$. major se fixent sur la galectine- 3 à la surface des macrophages, dont la réponse immunitaire est réprimée. La couche de galectine- 3 est alors détruite et la réponse immunitaire des macrophages peut être activée sous l'effet d'une stimulation externe. inflammatoires, se lie spécifiquement aux épitopes polygalactose du LPG de L. major [32]. Cette interaction spécifique pourrait s'avérer déterminante dans la réponse inflammatoire massive locale provoquée par $L$. major. En effet, la galectine-3, qui forme une couche à la surface du macrophage (Figure 5) pourrait être détruite par la protéase GP63 exprimée à la surface du parasite. Le clivage de la galectine-3 par L. major provoquerait une augmentation de la sensibilité des macrophages à des stimuli immunologiques, ce qui pourrait expliquer la réponse locale inflammatoire induite par L. major $[32,33]$. Les amastigotes de $L$. major semblent également interagir avec des lectines mais leur surface étant dépourvue de GP63, ils utilisent sans doute d'autres stratégies d'interaction avec les macrophages. D'autres récepteurs de l'interaction Leishmania/cellule hôte ont été identifiés. La lectine DC-SIGN des cellules dendritiques semble agir comme récepteur pour les amastigotes de L. pifanoi [34] et l'interaction avec $L$. donovani fait intervenir plusieurs types de récepteurs dont la lectine MR (mannose receptor), l'intégrine CR3 et le récepteur des immunoglobulines FCgR (Figure 6). II sera intéressant de voir si $d$ 'autres espèces et stades du parasite sont reconnus par ces récepteurs.

L'interaction entre Leishmania et les macrophages ou les cellules dendritiques induit une modulation importante de l'expression génique autant chez le parasite que dans la cellule cible. Nous avons récemment démontré que l'activation de la phosphotyrosine phosphatase SHP-I induite par l'infection par $L$. donovani est responsable de I'inactivation de JAK2, une tyrosine kinase jouant un rôle clé dans l'activation de certaines fonctions du macrophage par l'interféron gamma [35] (Figure 6). Cette inactivation altère les fonctions microbicides du macrophage, favorisant ainsi la survie et la division du parasite. Des études in vivo nous ont permis de confirmer le rôle pivot joué par SHP-1 dans le développement de la leishmaniose cutanée et viscérale [36]. Des analyses transcriptomiques de macrophages infectés par $L$. donovani ont démontré que plusieurs gènes du macrophage sont régulés négativement, et plusieurs autres positivement, lors de l'infection [37]. Nos résultats préliminaires suggèrent que SHP-l serait largement impliqué dans la régulation de l'expression d'un très grand nombre de gènes murins, ainsi que dans l'atténuation de la transcription de plus de 100 gènes codant pour diverses fonctions immunitaires, signalétiques et cellulaires au cours de l'infection par L. donovani.

En conclusion, la génomique comparative appliquée à Leishmania a d'ores et déjà contribué à élargir nos connaissances concernant les interactions patho- 
gène/hôte cellulaire et de nombreuses autres applications sont envisageables. Une approche protéomique pourrait s'avérer fort intéressante pour identifier les divers substrats avec lesquels SHP-1 interagit chez les phagocytes et permettre de mieux comprendre les mécanismes conduisant à son activation. Ceci pourrait ainsi conduire au développement d'inhibiteurs beaucoup plus sélectifs contre SHP-1. De fait, nous avons démontré que des inhibiteurs modulant l'activité des phosphotyrosine phosphatases sont un excellent moyen de contrer les leishmanioses cutanées et viscérales par potentialisation des événements microbicides protecteurs (monoxyde d'azote) et de la réponse inflammatoire [38]. Des études transcriptomiques plus exhaustives des macrophages, mais également de modèles animaux infectés avec plusieurs espèces de Leishmania, permettront de mieux cerner la réponse de l'hôte au parasite. Il devrait être possible par exemple de mesurer les variations d'expression des gènes du parasite, de la cellule hôte ou de l'animal qui sont induites lors de l'infection (Figure 3).

\section{Chimiorésistance}

L'étude de la résistance à l'antimoine chez Leishmania fait partie des priorités de l'Organisation Mondiale de la Santé (www.who.int/infections-disease report/ 2000). Les mécanismes de résistance à l'antimoine ont été particulièrement bien étudiés in vitro et semblent faire intervenir plusieurs gènes [39]. L'antimoine pentavalent est probablement réduit in vivo et actif sous la forme trivalente quoique le site de réduction (I'hôte ou le parasite) soit encore indéterminé. Chez les parasites résistants, l'antimoine pentavalent serait conjugué à une molécule appelée trypanothion, qui est un composé glutathionspermidine spécifique aux kinétoplastidae. La concentration de trypanothion est effectivement augmentée chez les parasites résistants à l'antimoine [40]. Ce conjugué est par la suite soit séquestré à l'intérieur d'une vacuole par un transporteur de type $A B C$ [41] soit expulsé à l'extérieur de la cellule. Nous avons montré que chez les parasites résistants à l'antimoine, il existe des mécanismes de résistance additionnels, mais les approches cellulaires et moléculaires traditionnelles se sont avérées infructueuses pour les caractériser [39]. Grâce à des puces à $A D N$ à faible densité portant une cinquantaine de gènes, nous avons pu identifier de nouveaux gènes différentiellement exprimés chez les mutants résistants mais qui semblent toujours faire partie de la voie métabolique du trypanothion (données non publiées). Avec des puces portant le génome complet du parasite, de nouveaux gènes impliqués dans la résistance seront peut-être caractérisés, à condition bien sûr que le mécanisme de résistance fasse intervenir des changements dans le niveau d'expression des gènes. Chez Leishmania, le phénomène de résistance est de fait fréquemment associé à des processus d'amplification génique.

Nous avons récemment réalisé l'analyse protéomique de plusieurs parasites résistants ([42] et données non publiées). Chez des mutants amastigotes de L.d. infantum résistants à l'antimoine, $0,5 \%$ des protéines sont



Figure 6. Mécanismes de subversion de la signalisation du macrophage par Leishmania donovani. L'interaction du parasite avec la lectine MR (mannose receptor), l'intégrine CR3 et le récepteur des immunoglobulines $F C \gamma R$ à la surface de la cellule hôte active la phosphotyrosine phosphatase SHP-1 ce qui a pour effet d'inhiber l'activité des kinases JAK2 et Erk1/2. Plusieurs fonctions du macrophage (par exemple la voie de production de monoxyde d'azote [NO]) normalement induites par l'interféron $\gamma($ IFN $\gamma$ ) sont alors inhibées. De plus, l'activation de la voie SHP-l altère directement l'activité de plusieurs facteurs de transcription (NF-KB, IRF-1, AP1). Enfin, un mécanisme SHP-1-indépendant, passant par la voie PKC, pourraitprovoquer la dégradation du facteur de transcription STATl. iNOS: inducible nitric oxyde synthase; IL-12: interleukine-12. 
soit exclusivement exprimées soit totalement réprimées chez les mutants et une proportion encore supérieure de protéines sont sur ou sous-exprimées chez le mutant. Ces approches permettront de mieux comprendre les mécanismes de résistance, mais aussi d'étudier la réponse aux drogues et de déterminer leurs cibles. Une fois les bases moléculaires de la résistance à l'antimoine pentavalent connues, il sera possible de développer des tests diagnostiques rapides qui pourront prévenir l'utilisation de molécules toxiques devenues inefficaces.

\section{Conclusions}

La mise au point de techniques de transfection chez Leishmania au début des années 1990 a permis de passer d'une recherche descriptive à une recherche plus expérimentale. La disponibilité de la séquence du génome devrait être un puissant stimulant pour une recherche innovante chez ce parasite. En effet, avec plus de la moitié des gènes en quête d'une fonction, les parasitologistes ne chômeront pas. L'inactivation d'un gène par recombinaison homologue est possible chez Leishmania mais reste laborieuse et limitée aux gènes non essentiels [43]. Chez T. brucei, cousin lointain du Leishmania, la technologie des ARN inhibiteurs (ARNi) a déjà permis l'étude de plusieurs dizaines de gènes [44]. Pour l'instant, malgré les nombreux efforts de la communauté scientifique, cette technologie ne semble pas fonctionner chez Leishmania. De nouvelles stratégies de génomique fonctionnelle devront donc être développées. La possibilité de cultiver le parasite sous différentes formes au cours de son développement facilite les études sur le parasite lui-même mais également l'étude des interactions hôtes-pathogènes. Le séquençage des génomes humain et murin permettra également d'analyser de façon globale les modifications de I'hôte induites par le parasite. Les nombreuses lignées cellulaires déficientes pour des molécules clés de la réponse inflammatoire, immunitaire ou signalétique constituent de puissants outils pour une meilleure compréhension des interactions Leishmania/macrophages. On peut espérer qu'un projet génome de Lutzomyia ou Phlebotomus voit le jour, qui permettra alors l'étude au niveau global des interactions hôtes-parasites lors du cycle de vie complet de Leishmania. Une meilleure compréhension de la biologie du parasite et de son interaction avec l'hôte est essentielle pour développer de nouveaux médicaments dont l'action facilitera l'élimination et/ou le contrôle de ce pathogène soit par action directe sur le parasite, soit par modulation de la réponse de l'hôte. $\diamond$

\section{SUMMARY}

Studies on the parasite Leishmania

in the post-genomic era

Leishmania is a protozoan parasite responsible for considerable morbidity worldwide. The pathologies caused by Leishmania infections are varying with the species. The ongoing determination of the Leishmania major genome sequence represents a milestone for Leishmania research. We discuss here the use of transcriptomics and proteomics to accelerate our understanding of key processes related to Leishmania biology. These two techniques should be useful to find genes and proteins that are expressed in a stage-specific manner and examples of the use of such techniques are provided. Both approaches will complement each others. Indeed, while a number of stage-specific genes have increased stable RNA levels, an even larger subset of the Leishmania amastigote genes are regulated at the level of translation. The availability of the Leishmania genome should also permit important advances in finding species-specific genes that could explain different pathologies. Functional genomic and proteomic approaches should also be useful for understanding the mechanisms of drug resistance in the parasite. The availability of both the Leishmania genome and of its human host or of the mouse animal model will facilitate large scale studies and increase our understanding of hostpathogen interactions. $\diamond$

\section{RÉFÉRENCES}

1. Fleischmann RD, Adams MD, White 0 , et al. Wholegenome random sequencing and assembly of Haemophilus influenzae Rd. Science 1995; 269 : 496-512.

2. Gardner MJ, Hall N, Fung $\varepsilon$, et al. Genome sequence of the human malaria parasite Plasmodium falciparum. Nature 2002; 419: 498-511.

3.Sacks D, Kamhawi S. Molecular aspects of parasite-vector and vector-host interactions in leishmaniasis. Annu Rev Microbiol 2001; 55 : 453-83.

4. Herwaldt BL. Leishmaniasis. Lancet 1999; 354: 1191-9. 
5. Magill AJ, GrogI M, Gasser RA, Jr., Sun W, Oster CN. Visceral infection caused by Leishmania tropica in veterans of Operation Desert Storm. N Engl J Med 1993; 328: 1383-7.

6. Britto C, Ravel C, Bastien P, et al. Conserved linkage groups associated with large-scale chromosomal rearrangements between Old World and New World Leishmania genomes. Gene 1998; 222: 107-17.

7. Myler PJ, Audleman L, deVos T, et al. Leishmania major Friedlin chromosome 1 has an unusual distribution of proteincoding genes. Proc Natl Acad Sci USA 1999; 96 : 2902-6.

8. Clayton $C \varepsilon$. Life without transcriptional control? From fly to man and back again. EMBO / 2002; 21 : 1881-8.

9. McKean PG, Denny PW, Knuepfer $\varepsilon$, Keen JK, Smith DF. Phenotypic changes associated with deletion and overexpression of a stage-regulated gene family in Leishmania. Cell Microbiol 2001; 3: 511-23.

10. Sereno D, Lemesre JL. Axenically cultured amastigote forms as an in vitro model for investigation of antileishmanial agents. Antimicrob Agents Chemother 1997; 41: 972-6.

11. Lamontagne J, Papadopoulou B. Developmental regulation of spliced leader RNA gene in Leishmania donovani amastigotes is mediated by specific

polyadenylation.

J Biol Chem 1999; 274 . 6602-9.

12. Wu $Y$, عl Fakhry $y$, Sereno $D$, Tamar S, Papadopoulou B. A new developmentally regulated gene family in Leishmania amastigotes encoding a homolog of amastin surface proteins. Mol Biochem Parasitol 2000; 110: 345-57.
13. Charest $H$, Zhang WW, Matlashewski G. The developmental expression of Leishmania donovani A2 amastigote-specific genes is post-transcriptionally mediated and involves elements located in the $3^{\prime}$ untranslated region. J Biol Chem 1996; 271: 17081-90.

14. Bellatin JA, Murray AS, Zhao M, McMaster WR.

Leishmania mexicana: identification of genes that are preferentially expressed in amastigotes. Exp Parasitol 2002; 100 : 44-53.

15. Schoolnik GK. Functional and comparative genomics of pathogenic bacteria. Curr Opin Microbiol 2002; 5: 20-6.

16. Rathod PK, Ganesan K, Hayward RE, Bozdech Z, DeRisi JL. DNA microarrays for malaria. Trends Parasitol 2002; 18: 39-45.

17. Diehl S, Diehl F, El-Sayed N, Clayton C, Hoheisel J. Analysis of stage-specific gene expression in the bloodstream and the procyclic form of Trypanosoma brucei using a genomic DNA-microarray. Mol Biochem Parasitol 2002; 123: 115.

18. Almeida $R$, Norrish $A$, Levick M, et al. From genomes to vaccines: Leishmania as a model. Philos Trans $R$ Soc Lond B Biol Sci 2002; 357: 5-11.

19. Beverley SM, Akopyants NS, Goyard S, et al. Putting the Leishmania genome to work: functional genomics by transposon trapping and expression profiling. Philos Trans $R$ Soc Lond B Biol Sci 2002; 357: 47-53.

20. Talaat AM, Howard ST, Hale W 4th, Lyons R, Garner H, Johnston SA. Genomic DNA standards for gene expression profiling in Mycobacterium tuberculosis. Nucleic Acids Res 2002; 30 : ع104.
21. Chu S, DeRisi J, Eisen M, et al. The transcriptional program of sporulation in budding yeast. Science 1998; 282: 699-705.

22. Boucher N, Wu Y, Dumas C, et al. A common mechanism of stageregulated gene expression in Leishmania mediated by a conserved $3^{\prime}$ untranslated region element. J Biol Chem 2002; 277 : 19511-20.

23. El Fakhry $Y$, Ouellette $M$, Papadopoulou B. A proteomic approach to identify developmentally regulated proteins in Leishmania infantum. Proteomics 2002; 2 . 1007-17.

24. Gygi SP, Rist B, Gerber SA, Turecek F, Gelb MH, Aebersold R. Quantitative analysis of complex protein mixtures using isotopecoded affinity tags. Nat Biotechnol 1999; 17: 994-9.

25. Washburn MP, Wolters D, Yates JR, 3rd. Large-scale analysis of the yeast proteome by multidimensional protein identification technology. Nat Biotechnol 2001; 19: 242-7.

26. Zhang WW, Matlashewski G. Characterization of the A2A2rel gene cluster in Leishmania donovani: involvement of $A 2$ in visceralization during infection. Mol Microbiol 2001; 39: 935-48.

27. Carlton JM, Angiuoli SV, Suh $B B$, et al. Genome sequence and comparative analysis of the model rodent malaria parasite Plasmodium yoelii yoelii. Nature 2002; 419: 512-9.

28. Kündig $C$, Haimeur $A$, Légaré $D$, Papadopoulou $B$, Ouellette M. Increased transport of pteridines compensates for mutations in the high affinity folate transporter and contributes to methotrexate resistance in the protozoan parasite Leishmania tarentolae. EMBO J 1999; 18: 2342-51.
29. Myler PJ, Beverley SM, Cruz AK, et al. The Leishmania genome project: new insights into gene organization and function. Med Microbiol Immunol (Berl) 2001; $190: 9-12$.

30. Turco SJ, Descoteaux A. The lipophosphoglycan of Leishmania parasites. Annu Rev Microbiol 1992; 46: 65-94.

31. Matte C, Olivier M. Leishmania-induced cellular recruitment during the early inflammatory response: modulation of proinflammatory mediators. J Infect Dis 2002; 185: 673-81.

32. Pelletier I, Sato S. Specific recognition and cleavage of galectin-3 by Leishmania major through species-specific polygalactose epitope. J Biol Chem 2002; 277 : 17663-70.

33. Sato S. Galectin as a molecule of danger signal, which could evoke immune response to infection. Trends Glycosci Glycotechnol 2003 (sous presse).

34. Colmenares M, Puig-Kroger A, Pello OM, Corbi AL, Rivas L. Dendritic cell (DC)specific Intercellular adhesion molecule 3 (ICAM-3)-grabbing nonintegrin (DC-SIGN, CD209), a C-type surface lectin in human $D C s$, is a receptor for Leishmania amastigotes. J Biol Chem 2002; 277 : 36766-9.

35. Blanchette J, Racette N, Faure R, Siminovitch KA, Olivier M. Leishmaniainduced increases in activation of macrophage SHP-l tyrosine phosphatase are associated with impaired IFN-gamma-triggered JAK2 activation. Eur J Immunol 1999; 29 : 3737-44. 
36. Forget $G$, Siminovitch $K A$, Brochu S, Rivest S, Radzioch D, Olivier M. Role of host phosphotyrosine phosphatase SHP-1 in the development of murine leishmaniasis. Eur」 Immunol 2001; 31 : 3185-96.

37. Buates S, Matlashewski G. General suppression of macrophage gene expression during Leishmania donovani infection. J Immunol 2001 ; $166: 3416-22$.

38. Matte C, Marquis JF, Blanchette J, et al.

Peroxovanadium-mediated protection against murine leishmaniasis: role of the modulation of nitric oxide. Eur J Immunol 2000; 30 . 2555-64.
39. Légaré D, Cayer S, Singh AK, Richard D, Papadopoulou B, Ouellette M. ABC proteins of Leishmania. J Bioenerg Biomembr 2001 33: 469-74.

40. Haimeur A, Brochu C, Genest P, Papadopoulou B, Ouellette M. Amplification of the $A B C$ transporter gene PGPA and increased trypanothione levels in potassium antimonyl tartrate (SbIII) resistant Leishmania tarentolae. Mol Biochem Parasitol 2000; 108: 131-5.
41. Légaré $D$, Richard $D$, Mukhopadhyay $\mathrm{R}$, et al. The Leishmania $\mathrm{ABC}$ protein PGPA is an intracellular metal-thiol transporter ATPase. J Biol Chem 2001; 276: 26301-7.

42. Drummelsmith J, Brochu V, Girard I, Messier N, Ouellette M. Proteome mapping of the protozoan parasite Leishmania and application to the study of drug targets and resistance mechanisms. Mol Cell

Proteomics 2003; 2 :

146-55.
43. Dumas C, Ouellette M,

Tovar J, et al. Disruption of the trypanothione

reductase gene of

Leishmania decreases its ability to survive oxidative stress in macrophages. EMBO J 1997; 16: 2590-8.

44. Ullu $\varepsilon$, Djikeng $A$, Shi $H$, Tschudi C. RNA

interference: advances and questions. Philos Trans $R$ Soc Lond B Biol Sci 2002; $357: 65-70$

TIRÉS À PART

M. Ouellette
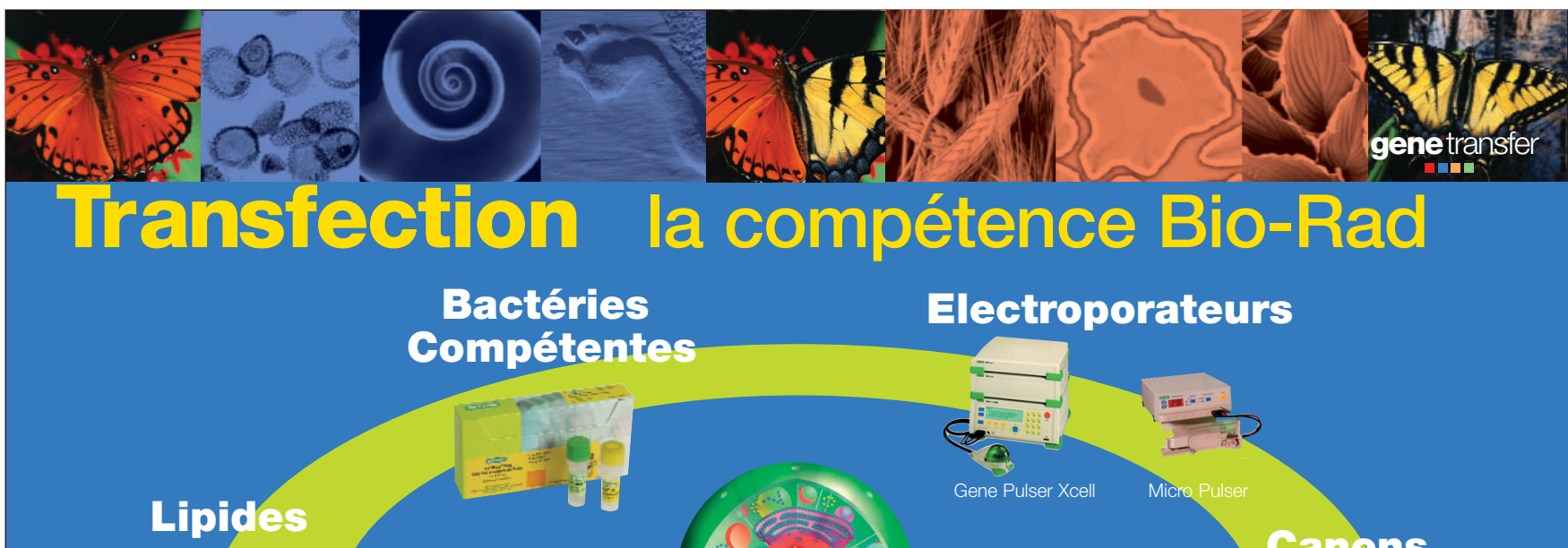

Bactéries

Electroporateurs

de Transfiection

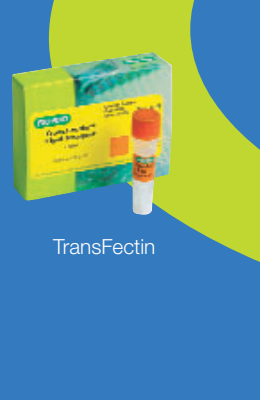

BIORAD

Bio-Rad 3, Boulevard Raymond Poincaré - 92430 Marnes-la-Coquette

Tél : 0147956965 • Fax : 0147956121 • E-mail : biorecherche@bio-rad.com 